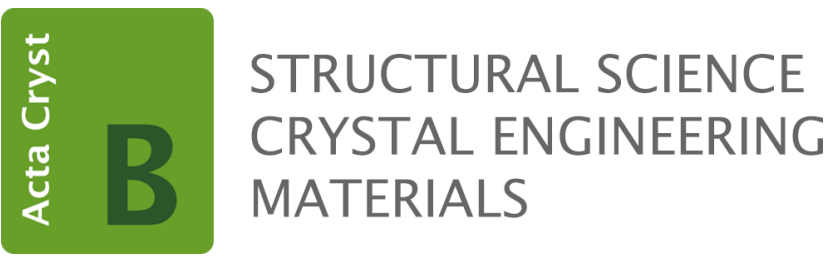

Volume 77 (2021)

Supporting information for article:

Low-temperature investigation of natural iron-rich oxoborates vonsenite and hulsite: thermal deformations of crystal structure, strong negative thermal expansion and cascades of magnetic transitions

Yaroslav P. Biryukov, Almaz L. Zinnatullin, Mikhail A. Cherosov, Andrey P. Shablinskii, Roman V. Yusupov, Rimma S. Bubnova, Farit G. Vagizov, Stanislav K. Filatov, M.S. Avdonceva and Igor V. Pekov 

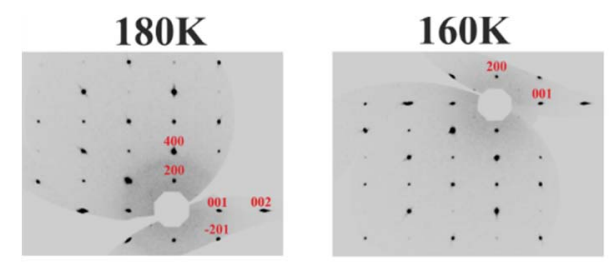

\section{$120 \mathrm{~K}$}

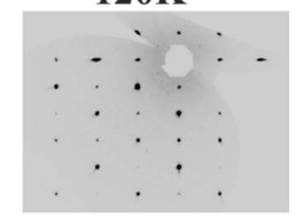

$100 \mathrm{~K}$

$80 \mathrm{~K}$
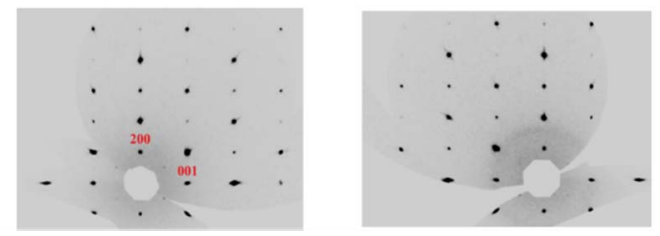

Figure S1 Reciprocal space for vonsenite at 80, 100, 120, 160 and $180 \mathrm{~K}$.

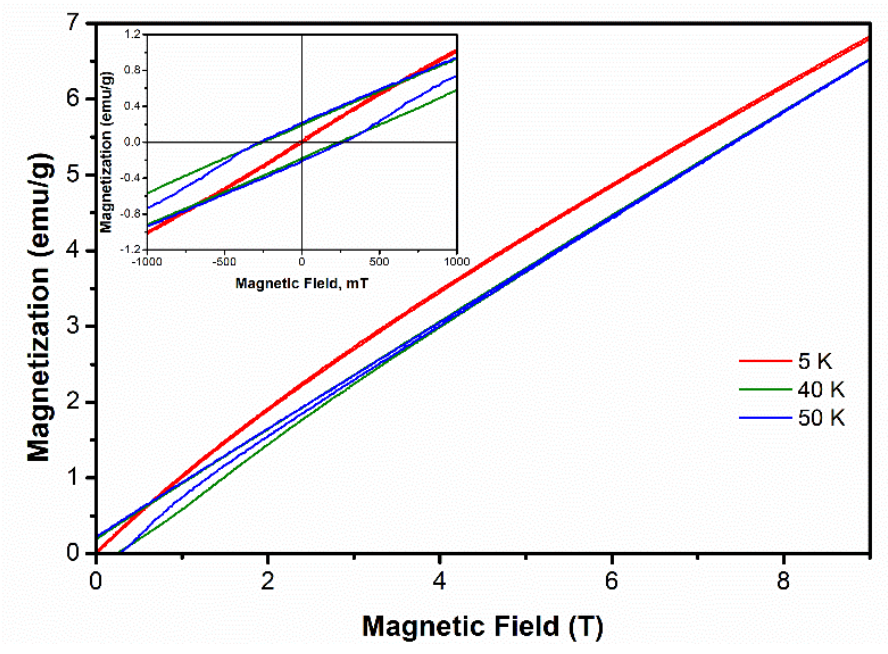

Figure S2 Field dependence of magnetization of hulsite recorded at 5, 40, and $50 \mathrm{~K}$. Inset is the data in the region of low magnetic field. 


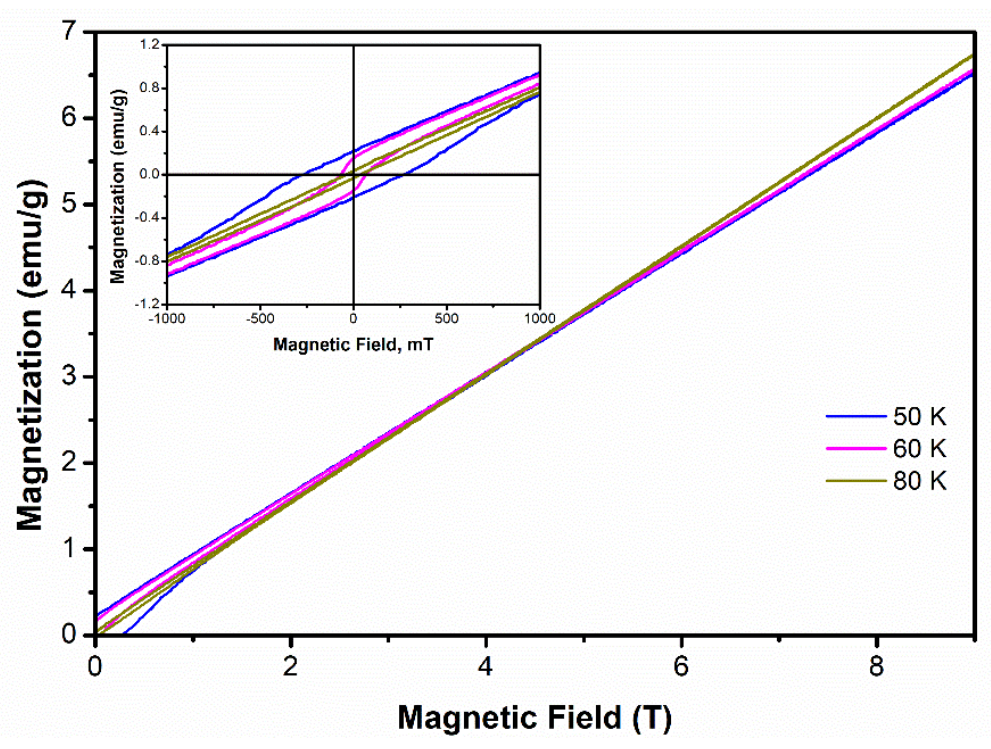

Figure S3 Field dependence of magnetization of hulsite recorded at 50, 60, and $80 \mathrm{~K}$. Inset is the data in the region of low magnetic field.

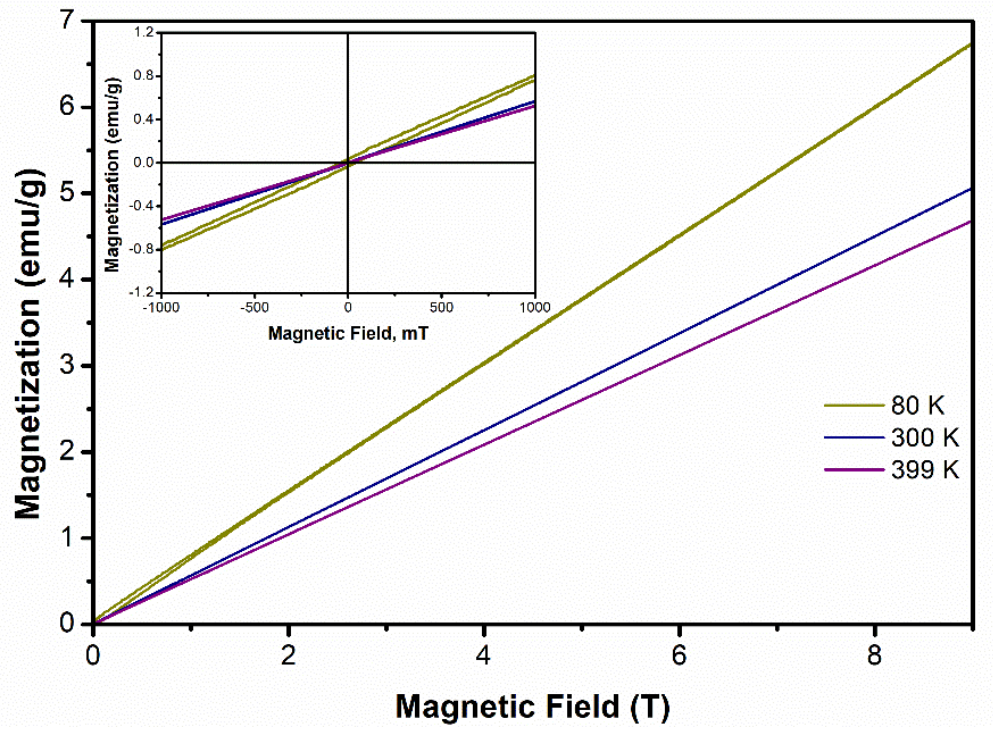

Figure S4 Field dependence of magnetization of hulsite recorded at 80,300 , and $399 \mathrm{~K}$. Inset is the data in the region of low magnetic field. 
Table S1 Fractional atomic coordinates and equivalent isotropic displacement parameters $\left(\AA^{2}\right)$ for hulsite and vonsenite $(100 \mathrm{~K})$.

\begin{tabular}{|c|c|c|c|c|c|}
\hline \multicolumn{6}{|c|}{ Hulsite } \\
\hline Atom & $x$ & $y$ & $z$ & $U_{\text {iso }} * / U_{\text {eq }}\left(\AA^{2}\right)$ & Occupancy \\
\hline $\mathrm{Fe} 1$ & 0 & 0 & 0 & $0.019(2)$ & $0.97(2)$ \\
\hline Sn1' & 0 & 0 & 0 & $0.019(2)$ & $0.03(2)$ \\
\hline $\mathrm{Fe} 2$ & 0 & 0.5 & 0.5 & $0.030(4)$ & $0.6(2)$ \\
\hline Mg2' & 0 & 0.5 & 0.5 & $0.030(4)$ & $0.4(2)$ \\
\hline $\mathrm{Fe} 3$ & 0.5 & 0 & 0 & $0.024(2)$ & 1 \\
\hline $\mathrm{Fe} 4$ & 0.5 & 0 & 0.5 & $0.021(2)$ & 1 \\
\hline $\mathrm{Fe} 5$ & $0.2726(4)$ & 0.5 & $0.2210(6)$ & $0.020(2)$ & 1 \\
\hline $\mathrm{O} 1$ & $-0.6908(16)$ & -1 & $-0.499(2)$ & $0.019(4)$ & 1 \\
\hline $\mathrm{O} 2$ & $0.4659(14)$ & 0.5 & $0.242(3)$ & $0.019(4)$ & 1 \\
\hline $\mathrm{O} 3$ & $-0.6969(16)$ & -1 & $-0.059(2)$ & $0.019(4)$ & 1 \\
\hline $\mathrm{O} 4$ & $-0.8872(13)$ & -1 & $-0.291(3)$ & $0.019(4)$ & 1 \\
\hline O5 & $0.0922(17)$ & 0.5 & $0.182(3)$ & $0.019(4)$ & 1 \\
\hline \multicolumn{6}{|c|}{ Vonsenite $(100 \mathrm{~K})$} \\
\hline Atom & $x$ & $y$ & $z$ & $U_{\text {iso }} * / U_{\text {eq }}\left(\AA^{2}\right)$ & Occupancy \\
\hline $\mathrm{Fe} 1$ & 0 & 0 & 0 & $0.0011(4)^{*}$ & 0.76 \\
\hline $\mathrm{Mg} 1^{\prime}$ & 0 & 0 & 0 & $0.0011(4)^{*}$ & 0.24 \\
\hline $\mathrm{Fe} 2$ & 0.5 & 0 & 0.5 & $0.0168(5)$ & 1 \\
\hline $\mathrm{Fe} 3$ & $0.0020(1)$ & $0.2760(1)$ & 0 & $0.0106(4)^{*}$ & 0.77 \\
\hline Mg3' & $0.0020(1)$ & $0.2760(1)$ & 0 & $0.0106(4)^{*}$ & 0.23 \\
\hline $\mathrm{Fe} 4$ & $0.7431(1)$ & $0.39731(8)$ & 0.5 & $0.0036(3)$ & 1 \\
\hline $\mathrm{O} 1$ & $0.8465(6)$ & 0.0439 (4) & 0.5 & $0.0059(10)^{*}$ & 1 \\
\hline $\mathrm{O} 2$ & $0.3871(6)$ & 0.0777 (4) & 0 & $0.0067(10)^{*}$ & 1 \\
\hline $\mathrm{O} 3$ & $0.6233(6)$ & $0.1410(4)$ & 0.5 & $0.0057(10)^{*}$ & 1 \\
\hline $\mathrm{O} 4$ & $0.1122(5)$ & $0.1410(4)$ & 0 & $0.0026(9)^{*}$ & 1 \\
\hline $\mathrm{O} 5$ & $0.3460(5)$ & $0.2622(4)$ & 0.5 & $0.0042(9)^{*}$ & 1 \\
\hline B1 & $0.2714(8)$ & $0.3606(6)$ & 0.5 & $0.0014(13)^{*}$ & 1 \\
\hline
\end{tabular}


Table S2 Anisotropic atomic displacement parameters $\left(\AA^{2}\right)$ of hulsite and vonsenite $(100 \mathrm{~K})$.

\begin{tabular}{|l|l|l|l|lll}
\hline Atom & $U_{11}$ & \multicolumn{1}{l}{$U_{22}$} & $U_{33}$ & $U_{13}$ & $U_{23}$ \\
\hline \multicolumn{7}{|c}{ Hulsite } \\
\hline $\mathrm{Fe} 1$ & $0.037(5)$ & $0.015(3)$ & $0.003(6)$ & 0 & $-0.001(2)$ & 0 \\
\hline $\mathrm{Sn} 1^{\prime}$ & $0.037(5)$ & $0.015(3)$ & $0.003(6)$ & 0 & $-0.001(2)$ & 0 \\
\hline $\mathrm{Fe} 2$ & $0.045(7)$ & $0.026(6)$ & $0.019(5)$ & 0 & $-0.005(3)$ & 0 \\
\hline $\mathrm{Mg} 2$ & $0.045(7)$ & $0.026(6)$ & $0.019(5)$ & 0 & $-0.005(3)$ & 0 \\
\hline $\mathrm{Fe} 3$ & $0.042(4)$ & $0.010(3)$ & $0.020(3)$ & 0 & $0.004(3)$ & 0 \\
\hline $\mathrm{Fe} 4$ & $0.024(4)$ & $0.024(4)$ & $0.015(3)$ & 0 & $0.000(3)$ & 0 \\
\hline $\mathrm{Fe} 5$ & $0.038(3)$ & $0.016(2)$ & $0.007(3)$ & 0 & $0.001(2)$ & 0 \\
\hline $\mathrm{O} 1$ & $0.031(8)$ & $0.018(7)$ & $0.006(8)$ & 0 & $-0.001(3)$ & 0 \\
\hline $\mathrm{O} 2$ & $0.031(8)$ & $0.018(7)$ & $0.006(8)$ & 0 & $-0.001(3)$ & 0 \\
\hline $\mathrm{O} 3$ & $0.031(8)$ & $0.018(7)$ & $0.006(8)$ & 0 & $-0.001(3)$ & 0 \\
\hline $\mathrm{O} 4$ & $0.031(8)$ & $0.018(7)$ & $0.006(8)$ & 0 & $-0.001(3)$ & 0 \\
\hline $\mathrm{O} 5$ & $0.031(8)$ & $0.018(7)$ & $0.006(8)$ & 0 & $-0.001(3)$ & 0 \\
\hline $\mathrm{Fe} 2$ & $0.0174(9)$ & $0.0146(9)$ & $0.0184(9)$ & $-0.0007(7)$ & 0 & 0 \\
\hline $\mathrm{Fe} 4$ & $0.0041(5)$ & $0.0037(5)$ & $0.0028(5)$ & $-0.0008(4)$ & 0 & 0 \\
\hline
\end{tabular}


Table S3 Selected bond lengths ( $\AA$ ) of hulsite and vonsenite (100 K).

\begin{tabular}{|c|c|c|c|c|c|}
\hline Bond & \multicolumn{2}{|c|}{ Distance ( $\AA$ ) BVS (v.u.) } & Bond & \multicolumn{2}{|c|}{ Distance $(\AA)$ BVS (v.u.) } \\
\hline \multicolumn{6}{|c|}{ Hulsite } \\
\hline $\mathrm{Fe} 1-\mathrm{O}^{\mathrm{i}}$ & $2.04(2)$ & 0.46 & $\mathrm{Fe} 4-\mathrm{O}^{\mathrm{vi}}$ & $2.03(2)$ & 0.46 \\
\hline $\mathrm{Fe} 1-\mathrm{O} 4^{\mathrm{ii}}$ & $2.04(2)$ & 0.46 & $\mathrm{Fe} 4-\mathrm{O} 1^{\mathrm{xii}}$ & $2.03(2)$ & 0.46 \\
\hline $\mathrm{Fe} 1-\mathrm{O} 5^{\mathrm{iii}}$ & $2.05(1)$ & 0.46 & $\mathrm{Fe} 4-\mathrm{O} 2^{\mathrm{iii}}$ & $2.10(1)$ & 0.38 \\
\hline $\mathrm{Fe} 1-\mathrm{O} 5$ & $2.05(1)$ & 0.46 & $\mathrm{Fe} 4-\mathrm{O} 2$ & $2.10(1)$ & 0.38 \\
\hline $\mathrm{Fe} 1-\mathrm{O} 5^{\text {iv }}$ & $2.05(1)$ & 0.46 & $\mathrm{Fe} 4-\mathrm{O} 2^{\text {xiii }}$ & $2.10(1)$ & 0.38 \\
\hline $\mathrm{Fe} 1-\mathrm{O}^{\mathrm{v}}$ & $2.05(1)$ & 0.46 & $\mathrm{Fe} 4-\mathrm{O} 2^{\text {xiv }}$ & $2.10(1)$ & 0.38 \\
\hline$<\mathrm{Fe} 1-\mathrm{O}>_{6}$ & 2.04 & 2.76 & $<\mathrm{Fe} 4-\mathrm{O}>{ }_{6}$ & 2.07 & 2.46 \\
\hline $\mathrm{Fe} 2-\mathrm{O} 4^{\mathrm{vi}}$ & $2.22(1)$ & 0.26 & $\mathrm{Fe} 5-\mathrm{O} 1^{\mathrm{vi}}$ & $2.18(1)$ & 0.30 \\
\hline $\mathrm{Fe} 2-\mathrm{O} 4^{\mathrm{vii}}$ & $2.22(1)$ & 0.26 & $\mathrm{Fe} 5-\mathrm{O} 1^{\mathrm{vii}}$ & $2.18(1)$ & 0.30 \\
\hline $\mathrm{Fe} 2-\mathrm{O} 4^{\mathrm{ii}}$ & $2.22(1)$ & 0.26 & $\mathrm{Fe} 5-\mathrm{O} 2$ & $2.05(2)$ & 0.43 \\
\hline $\mathrm{Fe} 2-\mathrm{O} 4^{\text {viii }}$ & $2.22(1)$ & 0.26 & $\mathrm{Fe} 5-\mathrm{O}^{\mathrm{i}}$ & $2.20(1)$ & 0.28 \\
\hline $\mathrm{Fe} 2-\mathrm{O} 5$ & $2.04(2)$ & 0.42 & $\mathrm{Fe} 5-\mathrm{O} 3^{\mathrm{xv}}$ & $2.20(1)$ & 0.28 \\
\hline $\mathrm{Fe} 2-\mathrm{O} 5^{\text {ix }}$ & $2.04(2)$ & 0.42 & $\mathrm{Fe} 5-\mathrm{O} 5$ & $1.92(2)$ & 0.60 \\
\hline$<\mathrm{Fe} 2-\mathrm{O}>{ }_{6}$ & 2.16 & 1.88 & $<\mathrm{Fe} 5-\mathrm{O}>{ }_{6}$ & 2.12 & 2.20 \\
\hline $\mathrm{Fe} 3-\mathrm{O} 2^{\mathrm{iii}}$ & $2.07(1)$ & 0.42 & $\mathrm{~B} 1-\mathrm{O} 1$ & $1.36(2)$ & 1.03 \\
\hline $\mathrm{Fe} 3-\mathrm{O} 2$ & $2.07(1)$ & 0.42 & $\mathrm{~B} 1-\mathrm{O} 3$ & $1.41(2)$ & 0.90 \\
\hline $\mathrm{Fe} 3-\mathrm{O} 2^{\mathrm{x}}$ & $2.07(1)$ & 0.42 & $\mathrm{~B} 1-\mathrm{O} 4$ & $1.37(2)$ & 1.00 \\
\hline $\mathrm{Fe} 3-\mathrm{O} 2^{\mathrm{xi}}$ & $2.07(1)$ & 0.42 & $<\mathrm{B} 1-\mathrm{O}>_{3}$ & 1.38 & 2.92 \\
\hline $\mathrm{Fe} 3-\mathrm{O}^{\mathrm{i}}$ & $2.10(2)$ & 0.38 & & & \\
\hline $\mathrm{Fe} 3-\mathrm{O} 3^{\mathrm{xii}}$ & $2.10(2)$ & 0.38 & & & \\
\hline$<\mathrm{Fe} 3-\mathrm{O}>_{6}$ & 2.08 & 2.43 & & & \\
\hline \multicolumn{6}{|c|}{ Vonsenite $(100 \mathrm{~K})$} \\
\hline $\mathrm{Fe} 1-\mathrm{O} 3$ & $2.077(6)$ & 0.41 & $\mathrm{Fe} 2-\mathrm{O} 4$ & $2.022(5)$ & 0.49 \\
\hline $\mathrm{Fe} 1-\mathrm{O} 3$ & $2.077(6)$ & 0.41 & $\mathrm{Fe} 2-\mathrm{O} 4$ & $2.022(5)$ & 0.49 \\
\hline $\mathrm{Fe} 1-\mathrm{O} 2$ & $2.084(4)$ & 0.40 & $\mathrm{Fe} 2-\mathrm{O} 1$ & $2.160(4)$ & 0.34 \\
\hline $\mathrm{Fe} 1-\mathrm{O} 2$ & $2.084(4)$ & 0.40 & $\mathrm{Fe} 2-\mathrm{O} 1$ & $2.160(4)$ & 0.34 \\
\hline $\mathrm{Fe} 1-\mathrm{O} 2$ & $2.084(4)$ & 0.40 & $\mathrm{Fe} 2-\mathrm{O} 1$ & $2.160(4)$ & 0.34 \\
\hline $\mathrm{Fe} 1-\mathrm{O} 2$ & $2.084(4)$ & 0.40 & $\mathrm{Fe} 2-\mathrm{O} 1$ & $2.160(4)$ & 0.34 \\
\hline$<\mathrm{Fel}-\mathrm{O}_{6}$ & 2.08 & 2.41 & $<\mathrm{Fe} 2-\mathrm{O}>_{6}$ & 2.11 & 2.34 \\
\hline
\end{tabular}




\begin{tabular}{|c|c|c|c|c|c|}
\hline $\mathrm{Fe} 3-\mathrm{O} 4$ & $1.949(6)$ & 0.50 & $\mathrm{Fe} 4-\mathrm{O} 4$ & $1.985(4)$ & 0.54 \\
\hline $\mathrm{Fe} 3-\mathrm{O} 2$ & $2.091(6)$ & 0.34 & $\mathrm{Fe} 4-\mathrm{O} 4$ & $1.985(4)$ & 0.54 \\
\hline $\mathrm{Fe} 3-\mathrm{O} 3$ & $2.155(4)$ & 0.29 & $\mathrm{Fe} 4-\mathrm{O} 5$ & $2.075(5)$ & 0.43 \\
\hline $\mathrm{Fe} 3-\mathrm{O} 3$ & $2.155(4)$ & 0.29 & $\mathrm{Fe} 4-\mathrm{O} 2$ & $2.076(5)$ & 0.42 \\
\hline $\mathrm{Fe} 3-\mathrm{O} 5$ & $2.159(4)$ & 0.28 & $\mathrm{Fe} 4-\mathrm{O} 2$ & $2.076(5)$ & 0.42 \\
\hline $\mathrm{Fe} 3-\mathrm{O} 5$ & $2.159(4)$ & 0.28 & $\mathrm{Fe} 4-\mathrm{O} 1$ & $2.091(6)$ & 0.41 \\
\hline$<\mathrm{Fe} 3-\mathrm{O}>_{6}$ & 2.11 & 1.98 & $<\mathrm{Fe} 4-\mathrm{O}>_{6}$ & 2.05 & 2.77 \\
\hline $\mathrm{B} 1-\mathrm{O} 3$ & $1.365(9)$ & 1.01 & & & \\
\hline $\mathrm{B} 1-\mathrm{O} 5$ & $1.380(10)$ & 0.97 & & & \\
\hline $\mathrm{B} 1-\mathrm{O} 1$ & $1.394(9)$ & 0.94 & & & \\
\hline$<\mathrm{B} 1-\mathrm{O}>_{3}$ & 1.38 & 2.92 & & & \\
\hline
\end{tabular}

BVS - bond valence sum

Table S4 Equations of approximation of temperature dependencies of unit cell parameters of vonsenite.

\begin{tabular}{lllll}
\hline \multirow{2}{*}{$\boldsymbol{T}(\mathbf{K})$} & \multicolumn{5}{l}{ Equation $\boldsymbol{l}(\boldsymbol{t})=\boldsymbol{l}_{\mathbf{0}}+\boldsymbol{l}_{\mathbf{1}} \boldsymbol{t}+\boldsymbol{l}_{\mathbf{2}} \boldsymbol{t}^{2}$} \\
\cline { 2 - 5 } & $\boldsymbol{a}(\boldsymbol{t})(\boldsymbol{\AA})$ & $\boldsymbol{b}(\boldsymbol{t})(\boldsymbol{\AA})$ & $\boldsymbol{c}(\boldsymbol{t})(\AA)$ & $\boldsymbol{V}(\boldsymbol{t})\left(\AA^{\mathbf{3}}\right)$ \\
\hline \multirow{3}{*}{$\mathbf{9 3 - 1 1 5}$} & $9.3771(1)-$ & $12.3791(2)-$ & $3.0555(3)+$ & $354.66(9)-$ \\
& $0.001221(2) \times t+$ & $0.00179(3) \times t+$ & $0.000043(6) \times t-$ & $0.0921(2) \times t+$ \\
& $0.0000052(1) \times t^{2}$ & $0.0000079(2) \times t^{2}$ & $0.00000016(3) \times t^{2}$ & $0.000404(8) \times t^{2}$ \\
\hline \multirow{3}{*}{$\mathbf{1 1 5}-\mathbf{3 1 3}$} & $9.3051(6)-$ & $12.2758(7)-$ & $3.0599(1)-$ & $349.53(3)-$ \\
& $0.000027(6) \times t+$ & $0.000016(7) \times t+$ & $0.000021(1) \times t-$ & $0.0039(4) \times t+$ \\
& $0.00000027(2) \times t^{2}$ & $0.00000028(2) \times t^{2}$ & $0.000000077(3) \times t^{2}$ & $0.000027(1) \times t^{2}$ \\
\hline
\end{tabular}

Table S5 Coefficients of approximation of temperature dependencies of unit cell parameters of hulsite.

\begin{tabular}{llllll}
\hline \multirow{2}{*}{$(\mathbf{K})$} & \multicolumn{5}{l}{ Equation $\boldsymbol{l}(\boldsymbol{t})=\boldsymbol{l}_{\mathbf{0}}+\boldsymbol{l}_{\boldsymbol{1}} \boldsymbol{t}+\boldsymbol{l}_{\boldsymbol{2}} \boldsymbol{t}^{\mathbf{2}}$} \\
\cline { 2 - 6 } & $\boldsymbol{a}(\boldsymbol{t})(\boldsymbol{\AA})$ & $\boldsymbol{b}(\boldsymbol{t})(\boldsymbol{\AA})$ & $\boldsymbol{c}(\boldsymbol{t})(\boldsymbol{\AA})$ & $\boldsymbol{\beta}(\boldsymbol{t})\left({ }^{\circ}\right)$ & $\boldsymbol{V}(\boldsymbol{t})\left(\AA^{\mathbf{3}}\right)$ \\
\hline \multirow{2}{*}{$\mathbf{9 3 - 1 2 0}$} & $10.6773(2)-$ & $3.0759(9)-$ & $5.4655(2)-$ & $93.635(3)+$ & $179.14(1)-$ \\
& $0.000173(3) \times t$ & $0.000052(9) \times t$ & $0.000091(2) \times t$ & $0.86(2) \times t$ & $0.0091(1) \times t$ \\
\hline \multirow{3}{*}{$\mathbf{1 2 0 - 3 9 0}$} & $10.6521(6)+$ & $3.0673(4)+$ & $5.4506(9)+$ & $93.778(9)-$ & $177.71(5)+$ \\
& $0.000016(6) \times t+$ & $0.000018(4) \times t+$ & $0.000028(8) \times t+$ & $0.51(9) \times t+$ & $0.0024(4) \times t+$ \\
& $0.00000018(3) \times t^{2}$ & $0.000000012(7) \times t^{2}$ & $0.000000032(1) \times t^{2}$ & $1.03(2) \times t^{2}$ & $0.0000047(8) \times t^{2}$ \\
\hline \multirow{3}{*}{$\mathbf{3 9 0 - 5 2 3}$} & $10.5721(5)+$ & $3.0781(1)-$ & $5.4506(9)+$ & $93.523(2)+$ & $176.41(2)+$ \\
& $0.000391(2) \times t-$ & $0.000033(5) \times t+$ & $0.000028(8) \times t+$ & $0.56(5) \times t$ & $0.0077(9) \times t-$ \\
& $0.00000026(2) \times t^{2}$ & $0.000000012(7) \times t^{2}$ & $0.000000077(6) \times t^{2}$ & & $0.0000011(1) \times t^{2}$ \\
\hline
\end{tabular}

